\title{
The Beneficial Effect of Enriched Environment on Pathogenesis of Alzheimer's Disease
}

\author{
Xingxing Chen ${ }^{* \#}$, Jie $\mathrm{Hu}^{2 *}$, Anbang Sun ${ }^{2 \#}$ \\ ${ }^{1}$ Department of Architecture, School of Urban Construction of Yangtze University, Jingzhou, China \\ ${ }^{2}$ Department of Anatomy, School of Basic Medical Sciences of Yangtze University, Jingzhou, China \\ Email: "35671106@qq.com, 1522474471@qq.com, "sunanbang327@163.com
}

How to cite this paper: Chen, X.X., Hu, J. and Sun, A.B. (2018) The Beneficial Effect of Enriched Environment on Pathogenesis of Alzheimer's Disease. Yangtze Medicine, 2, 225-243.

https://doi.org/10.4236/ym.2018.24025

Received: October 28, 2018

Accepted: December 18, 2018

Published: December 21, 2018

Copyright $\odot 2018$ by authors and Scientific Research Publishing Inc. This work is licensed under the Creative Commons Attribution International License (CC BY 4.0).

http://creativecommons.org/licenses/by/4.0/

\section{cc) (7) Open Access}

\begin{abstract}
Alzheimer's disease $(\mathrm{AD})$ is a common neurodegenerative disease, its main clinical symptoms are the progressive decline of cognitive and memory functions. Enriched Environment (EE) achieves the goal of improving brain cognitive reserve by enhancing the multi-directional stimulation on movement, sensory and cognitive systems of animals. And EE can regulate the levels of various trophic factors in the brain, promote synaptic regeneration and enhance neural plasticity to reduce the loss of neurons induced by inflammation. At present, there is still no effective treatment for $\mathrm{AD}$ and the clinical intervention drug is expensive. So it is essential to actively explore non-drug treatment. This review will explain the effects of EE on learning ability, memory ability and mental behavior in $\mathrm{AD}$, and provide a new direction for the treatment and rehabilitation of $\mathrm{AD}$.
\end{abstract}

\section{Keywords}

Alzheimer's Disease, Enriched Environment, Microtubule Associated Protein Tau, $\beta$-Amyloid Precursor Protein, Neurogenesis

\section{Introduction}

\subsection{Enriched Environment (EE)}

\subsubsection{The Origin and Concept of EE}

Enriched environment (EE) was first studied by Donald Hebb, who raised rats in his home and later showed that their performance of Morris water maze was superior compared with animals raised in laboratory [1]. Later, Rosenzweig et al. confirmed that EE can promote adult hippocampus neurogenesis and cognitive ability in rats [2] [3] [4]. EE was originally defined in 1978 as a complex of in-

*These authors have same contribution. 
animate objects and gregarious stimuli. Compared with Standard environment (SE), EE involves voluntary exercise, environmental enrichment, social interaction and spatial complexity, which are beneficial for improving cognitive reserve. The construction of EE is abundant and novel, which triggers the changes in growth factor synthesis, dendritic modifications, synaptic plasticity and neurogenesis [5] [6]. Horticultural therapy is the exercise of encouraging people to participate in planting or gardening activities to improve their bodies, minds, and spirits [7] [8]. In some ways, horticultural therapy is similar to EE, both of which provide patients with a more complex living environment, create a comfortable life to relieve the patient's mental stress and encourage patients to exercise more to enhance the various body systems.

\subsubsection{The Construction and Effect of EE}

Various forms of stimulation are the basic characteristics of EE, whose constructive principle is providing more chances for experiment animals to exercise and communicate. And EE is not only the copy of natural environment, but needs to be based on the living habits of the experiment animals, such as sleep habits and dietary habits. To promote the development of the behavior and the nervous system, it is important to provide experiment animals with a comfortable and relaxing living environment [9] [10] [11].

The construction of EE varies according to the aims of researchers and experimental animals. In general, EE cages are larger than that of SE. There is nothing except food and water in cages of SE. But there is a variety of sports equipment in EE cages, such as ladders, running wheels, platforms, tunnels, boxes, balls, blocks, swings, etc. (Figure 1). All of these are to ensure appropriate physical stimulation and spatial complexity, and encourage animals exercise voluntarily.

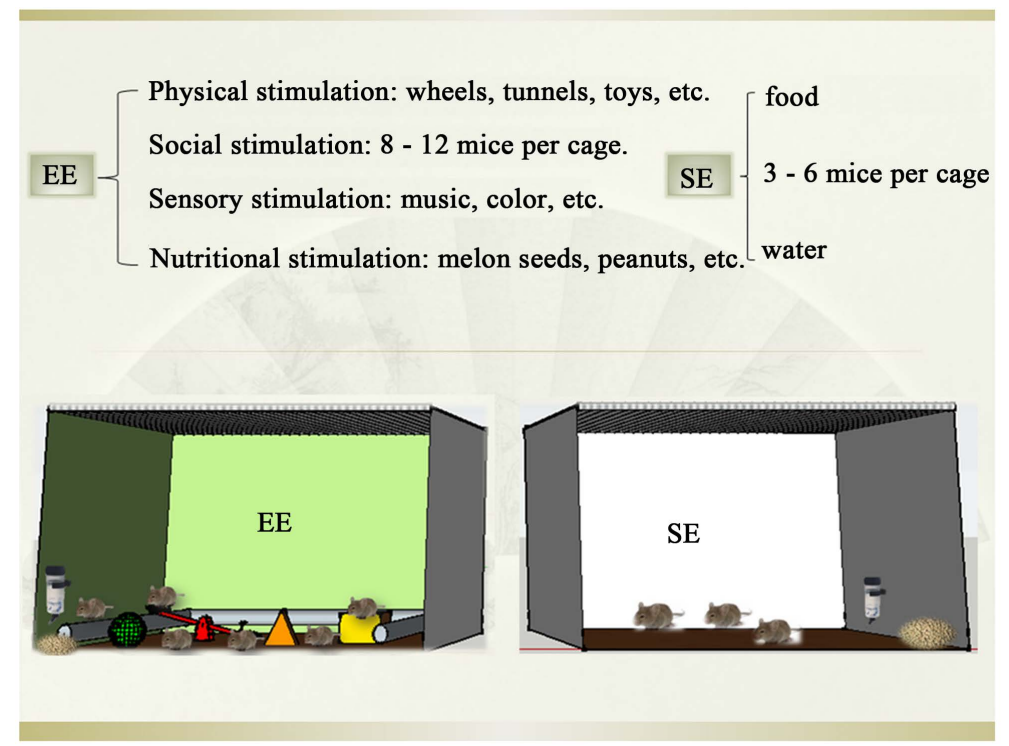

Figure 1. The construction of EE and SE. Compared with SE, EE is more complex, which contains running wheels, colorful tunnels, different toys and more animals except food and water. Abbreviations: EE, enriched environment; SE, standard environment. 
In order to maintain environmental novel, the layouts should be replaced 2 - 3 times a week. Some other forms of stimulations are added into experiment according to the research's needs, such as playing different types of music to promote animals' sensory perception and feeding more nutritive foods to promote animals' growth [12] [13] [14] [15] [16].

\subsubsection{Effects of EE on Neurogenesis and Cognitive Function in Normal Mice}

The EE has been widely used to explore the connection between cognition and neurogenesis since the concept of EE was proposed. Many studies have revealed EE can alter the morphology and molecular biology of animal brains [17] [18] [19] [20]. And EE can increase the number and volume of neuron in the visual cortex and hippocampus. Dendritic branches and density of dendritic spines are both increased to enhance neuronal plasticity and delay the decline of memory in aged rats [21] [22] [23].

As early as 1964, Altman et al. demonstrated that the glial cells in cortex of enriched rats increasing of 75 percent, which mainly differentiate into a large number of oligodendrocytes and a small amount of astrocytes [24]. In 1997, Epp et al. proposed that EE can increase the number of hippocampal neurons in adult mice and promote the survival of neuronal precursor cells in the hippocampal dentate gyrus (DG) [25] [26]. Later, Kempermann et al. confirmed that growth of old rats' hippocampal neurons was increased by 5 times and most of the new cells differentiated into astrocytes, when rats raised 10 to 20 months old under EE. That's to say, EE can induce neurogenesis, enhance the neural plasticity and decrease the aging rate of the nervous system even in middle-aged and old rats [27] [28] [29].

Emerging evidence have indicated that the expression of a variety of cellular growth factors are significantly increased in brain, which promote adult neurogenesis, such as brain-derived neurotrophic factor (BDNF), nerve growth factor (NGF), glial cell source, glial cell derived neurotrophic factor (GDNF), vascular endothelial growth factor (VEGF) and neurotrophin-3 (NT-3), etc. [30] [31] [32] [33]. And the EE animals could be less anxious in the presence of complex environment, because EE can lead neuronal endocrine changes and reduce the reactivity of the animal's hypothalamic-pituitary-adrenal axis (HPA axis) through regulating the neurotransmitter transmission of dopaminergic and glutamatergic neurons [34] [35]. Williams et al. demonstrated EE can promote the expression of calmodulin in hippocampus, which leads to increased phosphorylation of endogenous cAMP response element binding protein (CREB). And CREB is the key protein mediated spatial learning ability of memory and conducive to the consolidation of new memories [36] [37] [38]. Sharafi Z. et al. confirmed that noradrenergic neurons (NA) and serotonin (5-HT) neurons can be activated in the EE, which promote the release of monoamine neurotransmitters to enhance learning and synaptic plasticity in adult brains [39]. All of above factors are beneficial for brain's neurogenesis and improvement of neural plasticity. It also 
enhances the animal's learning and spatial memory ability to delay the progress of $\mathrm{AD}$.

\section{Alzheimer's Disease (AD)}

\subsection{The Concept and Clinical Symptoms of AD}

AD was discovered by German doctor Alois Alzheimer in 1906, which is one of the most common chronic degenerative diseases of the central nervous system. The main clinical symptoms are the progressive decline in cognitive and memory functions of patients, including various psychiatric symptoms and behavioral disorders [40]. With the aging of the global population, the incidence of $\mathrm{AD}$ in the world is increasing year by year. According to the research of World Alzheimer Reports 2018, there are 50 million people worldwide living with dementia and the number will increase to 152 million by 2050. In 2018, the cost of treating $\mathrm{AD}$ is estimated to exceed $\$ 1$ trillionand may reach $\$ 2$ trillion by 2030 [41]. AD seriously endangers the physical and mental health of the elderly and imposes a heavy burden on the society and the patients' family. It has become a serious social and medical problem.

\subsection{The Pathogenesis of AD}

The abnormal production and deposition of $\beta$-amyloid $(\mathrm{A} \beta)$ in senile plaques and hyper-phosphorylated Tau protein (p-Tau) in neurofibrillary tangles (NFTs) are hallmark features in the brains of $\mathrm{AD}$ patients. But pathological changes of $\mathrm{AD}$ are complex and diverse. There are also some other hypotheses about $\mathrm{AD}$, such as inflammatory, autophagy, oxidative stress, insulin signaling pathway dysfunction and mitochondrial dysfunction [42] [43] [44] (Figure 2).

\subsection{1. $\beta$-Amyloid}

$\mathrm{A} \beta$ is produced by the sequential cleavage of the amyloid precursor protein (APP) via a series of APP cleaving enzyme, such as $\alpha$-secretase, $\beta$-secretase and $\gamma$ secretase (Figure 2). The pathways of degradation of APP have both non-amyloidogic pathway (NAP) and amyloidogic pathway (AP) [45]. In NAP, APP is cleaved by $\alpha$-secretase to form soluble APP ( $\alpha$-sAPP), which is nutritive and protective for neurons. But in AP, APP is cleaved by $\beta$ and $\gamma$ secretase to form insoluble $\mathrm{A} \beta$ peptides, which are neurotoxic for neurons. Furthermore, $\alpha$-secretase appears to compete with $\beta$-secretase for the initial disintegration of APP, thus the expression and activity of both enzymes may affect the A $\beta$ level. In general, most APP is cleaved by $\alpha$-secretase, but the activity of $\beta$ and $\gamma$ secretase in $\mathrm{AD}$ patients is much higher than $\alpha$-secretase, leading to excessive insoluble $\mathrm{A} \beta$ deposition. And the common types of $\mathrm{A} \beta$ are $\mathrm{A} \beta 40$ and $\mathrm{A} \beta 42$. In normal conditions, APP is cleaved to produce a large amount $\mathrm{A} \beta 40$ and a small amount $\mathrm{A} \beta 42$. Although there is a little $\mathrm{A} \beta 42$, it's likely to deposit easily, because $\mathrm{A} \beta 42$ is more insoluble than $\mathrm{A} \beta 40$. Once metabolism of $\mathrm{A} \beta 42$ is abnormal, it is easy to deposit in the patients' brain to form neurotoxic SPs, induce dendrite and axon retraction and neuronal apoptosis [46] [47]. On the other hand, $\mathrm{A} \beta$ can activate 


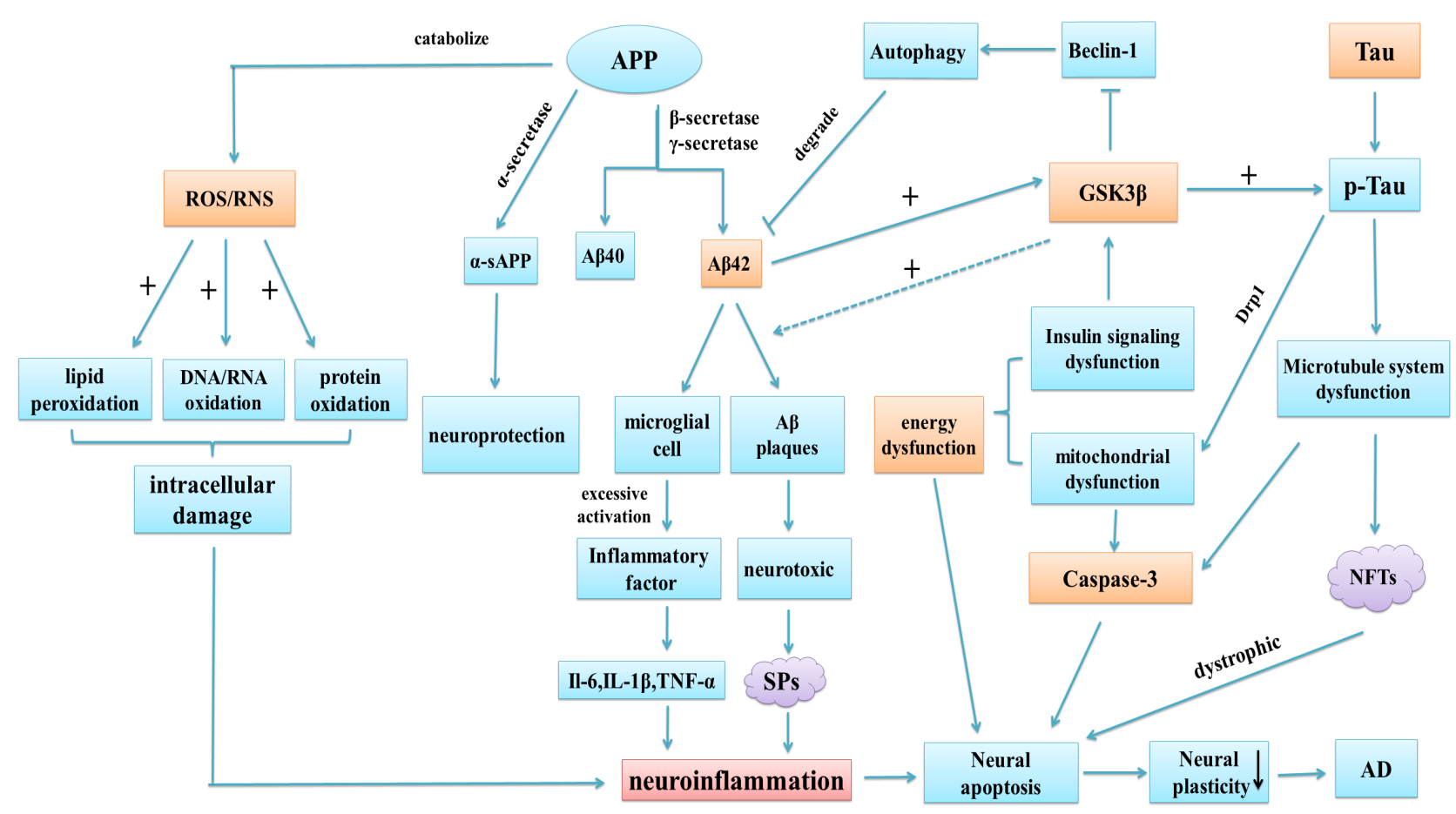

Figure 2. The major pathological mechanisms of AD. 1) SPs formed of insoluble A $\beta 42$ and NFTs formed of hyper-phosphorylated Tau can lead a large number of neuronal apoptosis induced by inflammation, which reduces the synaptic plasticity of neurons and exacerbates the occurrence of AD. 2) GSK- $3 \beta$ is the key enzyme that mediates A $\beta 42$ and p-Tau proteins, which regulates cell proliferation, survival and apoptosis by regulating multiple signaling pathways. 3) ROS/RNS can lead to the neuroinflammation through leading to the lipid peroxidation damage of cell membrane, destroying the oxidative modification of intracellular proteins, nuclear and mitochondrial DNA/RNA. In summary, multiple inflammatory pathways are involved in the development and progression of cognitive deficits in AD. Abbreviations: Drp1, dynamin related protein 1; Caspases, cysteine proteolytic enzymes; APP, amyloid precursor protein; p-Tau, hyper-phosphorylated Tau protein; SPs, senile plaques; NFTs, neurofibrillary tangles; $\alpha$-sAPP, soluble APP; ROS/RNS, reactive nitrogen species/reactive oxygen species.

monocytes and peripheral microglia via tyrosine kinase pathway to produce IL- $1 \beta$, IL-6, IL-8, TNF- $\alpha$ and other pro-inflammatory factors to trigger nervous system inflammatory immune response and accelerate neuronal apoptosis which directly lead to impairment of memory and cognitive [48] [49] [50] [51] [52].

\subsubsection{Tau Protein}

Tau protein is an important microtubule-associated protein expressed in neuron axons of the central nerve system, regulating the balance of phosphorylation and non-phosphorylation to keep the stability of the neuronal cytoskeleton. Researches have shown that main component of NFTs is abnormal p-Tau proteins. The clinical data also show that $\mathrm{p}$-Tau proteins in cerebrospinal fluid (CSF) are significantly elevated in $\mathrm{AD}$ patients [53] [54]. Thus, tangle formation and Tau release might contribute to the increased levels of Tau in CSF, suggesting that Tau level in CSF could be a good predictor of the early neurofibrillary changes occurring in brain of AD patients. And NFTs can reduce the affinity of microtubules, block the nutrient transport pathway of the microtubules and lead to atrophic apoptosis of neuronal dendrites and axons [55]. Moreover, $\mathrm{A} \beta \mathrm{can}$ improve contents of $\mathrm{p}$-Tau proteins and lead to an increase of NFTs, while Tau 
protein can also mediate neurotoxic lesions of $\mathrm{A} \beta$. Both of them accelerate the development of AD [56] [57] [58].

\subsubsection{Oxidative Stress}

The dynamic balance between production and elimination of reactive nitrogen species (RNS) and reactive oxygen species (ROS) is broken in body, leading to excessive accumulation of ROS. And ROS can not only directly lead to lipid peroxidation damage of cell membrane, but destroy the oxidative modification of intracellular proteins, nuclear and mitochondrial DNA/RNA (Figure 2). In the early stage, ROS can induce hyper-phosphorylation of Tau protein and production of $\mathrm{A} \beta$, indicating that ROS occurs earlier than the mild cognitive impairment, SPs and NFTs, thus to lead to cell and tissue damages and promote the disease progression. In addition, inflammatory responses induced by ROS also directly lead to neuronal necrosis or apoptosis [59] [60] [61].

\subsubsection{Autophagy}

Autophagy is a highly primitive protective mechanism mediated by autophagy associated genes (ATG) to maintain intracellular homeostasis [62] [63]. Varo et al. have shown that many autophagic vesicles are accumulated in the $\mathrm{A} \beta$ plaques, while few autophagic vesicles exist in normal brain tissue, indicating that the clearance of $\mathrm{A} \beta$ is associated with the level of autophagy [64]. Beclin-1 is an important regulator in the formation of autophagic vesicles, decreased expression of which not only affects the process of phagocytosis, but also reduces the cleared ability of microglia (Figure 2). In addition, the stable microtubule system is essential to transport autophagic vacuoles to lysosomes efficiently, which ensures the normal function of autophagy. However, hyper-phosphorylated Tau protein loses the ability to correctly bind to microtubules of neurons, making autophagosomes unable to bind to tubulin and transport to lysosome, thus, leading to autophagy dysfunction [65] [66] [67]. This may indicate that autophagy can protect the brain's neurogenesis and cognitive function by removing abnormally accumulated proteins in the cell.

\subsection{Current Treatments of $A D$}

At present, the common drugs of treatment for $\mathrm{AD}$ are cholinergic drugs, neuroprotective drugs, antioxidants, non-steroidal anti-inflammatory drugs (NSAIDs) and drugs for improving cerebral circulation or brain metabolism [68] [69] [70] [71]. Up to now, acetylcholinesterase inhibitors are the most effective drugs, which can inhibit the activity of acetylcholinesterase to reduce the breakdown of acetylcholine in the brain [72]. Monoclonal antibody vaccines against $\mathrm{A} \beta$ and Tau proteins have been studied popularly in the past decades, and have achieved remarkable results in animal experiments. Unfortunately, major pharmaceutical companies have failed in phase III clinical trials, despite the inoculation of $\mathrm{A} \beta$ antibodies can reduce the content of $\mathrm{A} \beta$ in the brain, but it does not improve patients' cognitive impairment, and even bring huge side effects to patients [73] 
[74] [75] [76] [77]. Therefore, the vaccine research on $A \beta$ and Tau protein has fallen into a bottleneck. How to improve the specificity of $\mathrm{A} \beta$ antibody and evade the adverse effects of vaccine on the autoimmune system will become the future research direction. Another popular research topic is autophagy, as a widely existed and conserved protective mechanism in cells, playing an important role in regulating clearance of $\mathrm{A} \beta$ plaques and $\mathrm{p}$-Tau protein [78] [79]. Therefore, future's research on autophagy regulators has provided a new direction for the treatment of $\mathrm{AD}$ [80]. Furthermore, the research of earlier non-drug treatment methods (rehabilitative garden therapy and horticultural therapy) has become a hot topic in current research because of the clinical drugs are expensive and the effect is not good. The research of EE on AD has also been developed gradually in China in recent decades [81].

\section{The Applications of EE}

\subsection{The Applications of EE in AD Mice}

\subsubsection{The Effect of EE on $A \beta$}

The SPs formed of excessive $\mathrm{A} \beta$ plaques is the typical pathological feature of $\mathrm{AD}$. Therefore, how to efficiently degrade and alleviate the accumulation of $\mathrm{A} \beta$ becomes an important method of treating AD. The NAP is the main pathway for the normal hydrolysis of APP in the brain, while $\alpha$-secretase is an important hydrolase which mediated the pathway of NAP hydrolysis. Hence, the APP in the brain will not hydrolyze normally and abnormal hydrolysis pathway will be activated by $\alpha$-secretase abnormal. Furthermore, ADAM9, ADAM10 and ADAM17 are three key genes to regulate $\alpha$-secretase, which play important roles in the normal hydrolysis of APP, and ADAM10 directly exerts the $\alpha$-secretase site degradation of APP. The expression of ADAM10 was decreased in hippocampus of APP transgenic mice, suggesting that it may be one of the important reasons for the excessive accumulation of APP in the brain [82] [83]. And researches have indicated that expression of ADAM10 increased significantly in the hippocampal of APP transgenic mice via chronic running exercise in EE for five months $(\mathrm{P}<0.05)$. This indicate that ADAM10 may promotes the normal hydrolysis of APP and significantly reduces the content of insoluble A $\beta 42$, thus to reduce its excessive deposition [84]-[90] (Figure 3). Mainardi et al. [91] and Maesako et al. [92] demonstrated that $\mathrm{EE}$ can reduce the $\mathrm{A} \beta$ plaques in the brain of aging rats by increasing the expression of $A \beta$-degrading enzymes. Li and his college also confirmed that $\mathrm{EE}$ can significantly reduce the contents of $\mathrm{A} \beta$ in hippocampal CA1 region of SAMP8 mice and effectively reduce neuronal apoptosis [93].

\subsubsection{The Effect of EE on Protein Tau}

Tau protein plays an important role in the stabilization of the microtubule system of neurons. The NFTs which formed by hyper-phosphorylated Tau protein accumulated in cerebral cells is the significant pathological feature of AD. Lazarov et al. [94] demonstrated that $\mathrm{EE}$ can reduce $\mathrm{A} \beta$ levels and amyloid deposition 


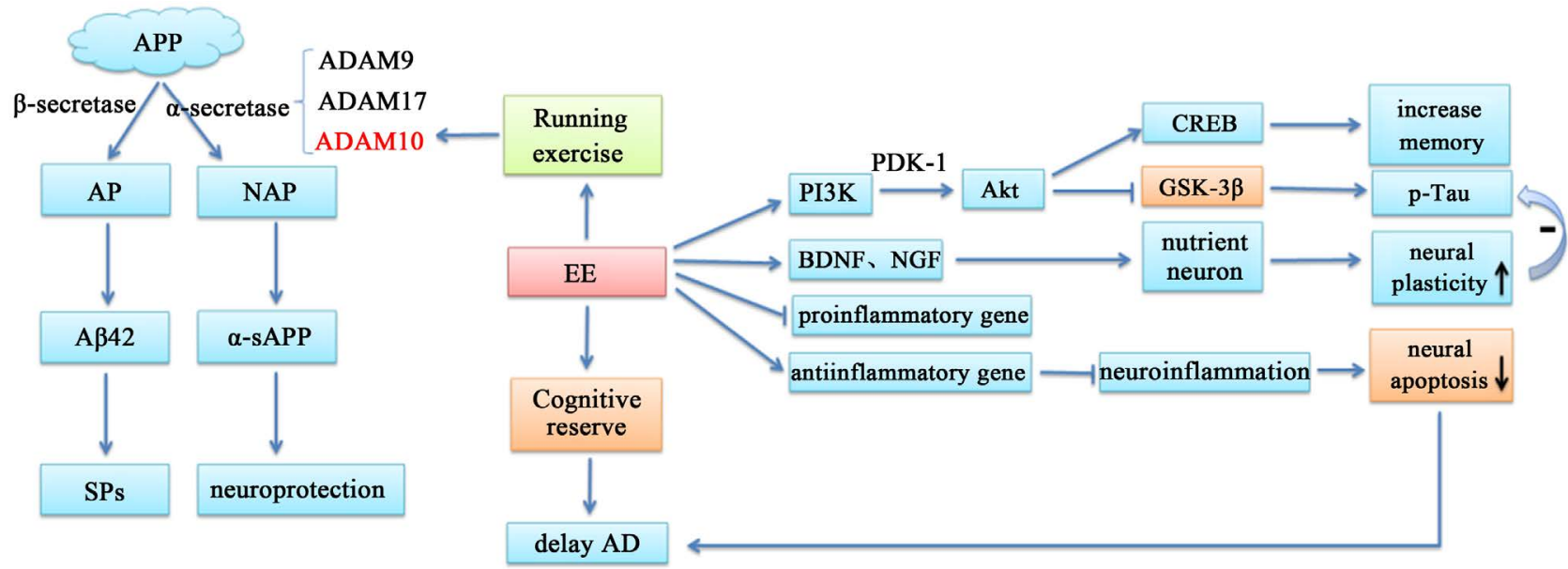

Figure 3. The mechanisms of EE on AD mice. 1) EE is beneficial for improving animals' cognition reserve to attenuate pathologic symptoms in Alzheimer's disease. 2) Long-term exposure in EE, proliferation of hippocampal cells is enhanced significantly. 3) Voluntary chronic exercise in EE activates the genes that mediated the degradation of APP. 4) EE rescues impaired neurogenesis and enhances synaptic plasticity by promoting the expression of various neuronal nutrient factors and regulating PI3K signal pathway. Abbreviations: CREB: cAMP response element binding protein; Akt/PKB: protein kinase B; PI3K: phosphatidylinositol kinase-3; NAP: non-amyloidogic pathway; AP: amyloidogic pathway; SPs: senile plaques.

in Transgenic Mice. Hu et al. [95] have shown that Tau transgenic mice have lower phosphorylated Tau protein in the EE compared with SE. In addition, the expression of BDNF in enriched group is significantly higher than that in standard group, which means that BDNF is likely to participate in decreased expression of Tau protein [96]. And chronic endurance exercise can enhance the antioxidant enzyme system of Tau transgenic mice, increase the expression of a series of antioxidant enzymes such as catalase, and activate phosphatidylinositol kinase-3 (PI3K). At the same time, PI3K can activate PDK-1, an activator of the upstream pathway of serine/threonine-protein kinase (Akt/PKB), to activate the enzymatic activity of Akt, while Akt can inhibit the hyper-phosphorylation of Tau via inhibiting the activity of GSK-3 $\beta$ in the downstream pathway [97] [98] (Figure 3).

\subsubsection{The Effect of EE on Neural Plasticity}

Complex environment is beneficial for activating voluntary exercise, increasing cognitive reserve and promoting proliferation and differentiation of hippocampal neonatal neural precursor cells in AD mice (Figure 3). Many researchers have indicated that APP transgenic mice in EE have lower levels of phosphorylated Tau protein in enriched group than standard group, which is associated with increased expression of a series of trophic factors such as BDNF, VEGF and NGF in APP transgenic mice. And the trophic factors can enhance protection and regeneration of neurons, promote the proliferation and phagocytic level of glial cells to improve the axonal transport, which is useful for degradation of $\mathrm{A} \beta$ plaques and $\mathrm{p}$-Tau [99] [100]. Furthermore, EE reduces the expression of pro-inflammatory genes while increasing the expression of anti-inflammatory genes, greatly reducing the level of neuronal apoptosis induced by inflammation 
[101] [102]. In short, long-term exposure in EE, proliferation of hippocampal cells is enhanced significantly and matured to become new neurons and glia, while enhanced neurogenesis was accompanied by a significant reduction in levels of hyper-phosphorylated Tau and $\mathrm{A} \beta$ plaques.

\subsection{The Application of EE in AD Patients}

In recent decades, a large number of animal experiments are shown that EE has a good effect on the emotional, mental behavior and cognitive memory in $\mathrm{AD}$ animals. Therefore, some hospitals began to combine with the constructive principles of EE to establish a comprehensive rehabilitation garden, which provide $\mathrm{AD}$ patients with a variety of simple games and exercise methods to improve patients' living ability, such as Chongqing Ge-Le-Shan Geriatric Rehabilitation Hospital. Music therapy, exercise therapy, planting flowers, jigsaw puzzles and other simple rehabilitation methods are used to encourage patients to do exercises. It is found that the cognitive function, mood and living ability of patients can be improved significantly, via various comprehensive exercises in the EE. In short, the main role of EE is to increase the brain's cognitive reserve by constantly trying new things, to promote brain's neurogenesis and enhance neuronal plasticity, thus to delay the decline of cognitive function [103] [104] [105].

\subsection{The Application of EE in Other Neurodegenerative Diseases}

The improvement of EE on neurodegenerative diseases was first discovered in Huntington's disease (HD) [106]. Researches indicated that EE can delay the atrophy of the brain and the onset of HD symptoms, and restore the neuronal regeneration function of SVZ region [107]. The study of EE on transient whole-cerebral ischemia rats and $\mathrm{AD}$ mouse model also found that the sensory movement, learning ability and memory function were enhanced after enriched stimulations. And the secretion of NGF, BDNF, GDNF and other nutrient factors increased significantly than the standard group, which means that neuronal regeneration may be associated with neurotrophic factors [108] [109]. Furthermore, compared with mice grown in the SE, the visual acuity of mice which long-term lived in EE increased by $18 \%$, suggesting that $\mathrm{EE}$ is conducive to the development of the visual system [110]. EE can also improve non-spatial memory defects in N-methyl-D-aspartate receptor knockout mice and promote cognitive and spatial memory in aged rats via enhancing glutamate decarboxylase activity and hippocampal synaptic density [111] [112] [113]. In addition, EE has a positive effect on the recovery of depression, Parkinson's disease (PD) and other neurological diseases [114] [115] [116] [117].

\section{Conclusions}

Although morbidity of $\mathrm{AD}$ is very high in the old people, it may have produced unknown pathological changes at the early stage, which means that $\mathrm{AD}$ may be slowly formed over a long period of time. When obvious AD symptoms are 
found, it is generally beyond the scope of drug intervention to reach an irreversible level. Due to the large individual differences and the difference between the exogenous input of $\mathrm{A} \beta$ and the $\mathrm{A} \beta$ formed pathologically, it has brought great difficulties to the modeling of $\mathrm{AD}$, which directly leads to the difference between actual pathological situation and the model. Hence, the results of the study are not applicable to clinical research, which may be an important reason why the research on $\mathrm{AD}$ has not made substantial progress over the years. As one of the emerging rehabilitation methods, $\mathrm{EE}$ has been proven to promote the secretion of various neurotrophic factors and improve neurogenesis in the study of neurological diseases, such as cerebral ischemia and hypoxic brain damage, craniocerebral trauma, $\mathrm{PD}$ and $\mathrm{AD}$. Although some researchers' experimental results did not achieve the expected results, this may be related to the differences in established models and environmental settings. There are still many needs to be improved based on the current research on EE. For example, environmental setting, interventional optimal time, intensity, age range and duration for different groups of people need to be further explored.

In addition, SPs formed of $\mathrm{A} \beta$ deposition and NFTs formed of $\mathrm{p}$-Tau in brain tissue induce different degrees of neuronal inflammation in AD patients. The immune response induced by inflammation accelerates aging and apoptosis of neurons, but long-term living in EE can increase cognitive reserve, promote the secretion of a variety of neurotrophic factors and enhance synaptic reconstruction after enriched stimulations. Therefore, EE can rescue impaired neurogenesis and enhance synaptic plasticity efficiently to delay the rate of degeneration of the nervous system and improve cognitive impairment. In future studies, we should explore the inflammatory mechanism and neuro-immune response mechanism of $\mathrm{EE}$ on $\mathrm{AD}$, and $\mathrm{EE}$ will play a major role in the treatment and recovery of AD.

\section{Acknowledgements}

The study was supported by Medical and Health Science and Technology Projects of Jingzhou Science and Technology Bureau (No.JZKJ18087), Guiding Projects of Science and Technology Research Program of Hubei Provincial Department of Education (B2018036), the Hubei Province Health and Family Planning Scientific Research Project (WJ2018H191), the Yangtze Youth Fund (NO.2015cqn79), the Yangtze Fund for Youth Teams of Science and Technology Innovation (NO.2016cqt04), Student Innovation Training Program of Yangtze University Health Science Center (ydc2017004, 2017006).

\section{Conflicts of Interest}

The authors have no conflicts of interest to disclose.

\section{References}

[1] Hebb, D.O. (1947) The Effects of Early Experience on Problem-Solving at Maturity. American Psychologist, 2, 737-745. 
[2] Rosenzweig, M.R. and Bennett, E.L. (1996) Psychobiology of Plasticity: Effects of Training and Experience on Brain and Behavior. Behavioural Brain Research, 78, 57-65. https://doi.org/10.1016/0166-4328(95)00216-2

[3] Rosenzweig, M.R., Bennett, E.L. and Diamond, M.C. (1972) Brain Changes in Response to Experience. Scientific American, 226, 22-29.

https://doi.org/10.1038/scientificamerican0272-22

[4] Nilsson, M., Perfilieva, E., Johansson, U., Orwar, O. and Eriksson, P.S. (1999) Enriched Environment Increases Neurogenesis in the Adult Rat Dentate Gyrus and Improves Spatial Memory. Journal of Neurobiology, 39, 569-578. https://doi.org/10.1002/(SICI)1097-4695(19990615)39:4<569::AID-NEU10>3.0.CO; $\underline{2-\mathrm{F}}$

[5] Stern, Y. (2002) What Is Cognitive Reserve? Theory and Research Application of the Reserve Concept. Journal of the International Neuropsychological Society, 8, 448. https://doi.org/10.1017/S1355617702813248

[6] Van, P.H., Kempermann, G. and Gage, F.H. (2000) Neural Consequences of Environmental Enrichment. Nature Reviews Neuroscience, 1, 191-198. https://doi.org/10.1038/35044558

[7] Blake, M. and Mitchell, G. (2016) Horticultural Therapy in Dementia Care: A Literature Review. Nursing Standard, 30, 41-47. https://doi.org/10.7748/ns.30.21.41.s44

[8] Wu, H.-Y., et al. (2018) Effect of Horticultural Therapy on Cognitive Function and Quality of Life in Patients with Mild-to-Moderate Alzheimer Disease. Chinese Journal of Multiple Organ Diseases in the Elderly, 3, 197-201.

[9] Will, B., et al. (2004) Recovery from Brain Injury in Animals: Relative Efficacy of Environmental Enrichment, Physical Exercise or Formal Training (1990-2002). Progress in Neurobiology, 72, 167-182. https://doi.org/10.1016/j.pneurobio.2004.03.001

[10] Rosenzweig, M.R., et al. (1978) Social Grouping Cannot Account for Cerebral Effects of Enriched Environments. Brain Research, 153, 563-576. https://doi.org/10.1016/0006-8993(78)90340-2

[11] Jankowsky, J.L., et al. (2005) Environmental Enrichment Mitigates Cognitive Deficits in a Mouse Model of Alzheimer's Disease. Journal of Neuroscience, 25, 5217-5224. https://doi.org/10.1523/JNEUROSCI.5080-04.2005

[12] Baumans, V. (2005) Environmental Enrichment for Laboratory Rodents and Rabbits: Requirements of Rodents, Rabbits, and Research. Ilar Journal, 46, 162-170. https://doi.org/10.1093/ilar.46.2.162

[13] Wang, Z.X., et al. (2003) The Effect of Music on Spatial Memory of Rat. Chinese Journal of Behavioral Medical Science, 12, 622-623.

[14] Hu, J.J., Wang, Z., et al. (2007) Preliminary Studies of Spacial Learning and Memory and Its Mechanism of Mice in Different Music Circumstance. Journal of Jinan University (Medicine Edition), 28, 132-135.

[15] Liu, Y.Y., et al. (2004) Effects of Different Light-Dark Cycle on Learning and Memory in Mice. Space Medicine \& Medical Engineering, 17, 381-382.

[16] Cao, L., et al. (2008) The Effect of Odors on Learning, Memory and Intracerebral Acetylcholinesterase Activity of the Mice. Chinese Journal of Gerontology, 28, 1465-1467.

[17] Nithianantharajah, J. and Hannan, A.J. (2006) Enriched Environments, Experience-Dependent Plasticity and Disorders of the Nervous System. Nature Reviews Neuroscience, 7, 697-709. https://doi.org/10.1038/nrn1970 
[18] Singh, P., Heera, P.K. and Kaur, G. (2003) Expression of Neuronal Plasticity Markers in Hypoglycemia Induced Brain Injury. Molecular \& Cellular Biochemistry, 247, 69-74. https://doi.org/10.1023/A:1024105120087

[19] Schrijver, N.C., Pallier, P.N., Brown, V.J. and Würbel, H. (2004) Double Dissociation of Social and Environmental Stimulation on Spatial Learning and Reversal Learning in Rats. Behavioural Brain Research, 152, 307-314. https://doi.org/10.1016/j.bbr.2003.10.016

[20] van Praag, H., Kempermann, G. and Gage, F.H. (1999) Running Increases Cell Proliferation and Neurogenesis in the Adult Mouse Dentate Gyrus. Nature Neuroscience, 2, 266-270. https://doi.org/10.1038/6368

[21] Leal-Galicia, P., Castañeda-Bueno, M., Quiroz-Baez, R. and Arias, C. (2008) Long-Term Exposure to Environmental Enrichment Since Youth Prevents Recognition Memory Decline and Increases Synaptic Plasticity Markers in Aging. Neurobiology of Learning \& Memory, 90, 511-518. https://doi.org/10.1016/j.nlm.2008.07.005

[22] Arne, H., et al. (2009) Environmental Enrichment Enhances Cellular Plasticity in Transgenic Mice with Alzheimer-Like Pathology. Experimental Neurology, 216, 184-192. https://doi.org/10.1016/j.expneurol.2008.11.027

[23] Arnaiz, S.L., et al. (2004) Enriched Environment, Nitric Oxide Production and Synaptic Plasticity Prevent the Aging-Dependent Impairment of Spatial Cognition. Molecular Aspects of Medicine, 25, 91-101. https://doi.org/10.1016/j.mam.2004.02.011

[24] Altman, J. and Das, G.D. (1964) Autoradiographic Examination of the Effects of Enriched Environment on the Rate of Glial Multiplication in the Adult Rat Brain. Nature, 204, 1161-1163. https://doi.org/10.1038/2041161a0

[25] Epp, J.R., Spritzer, M.D. and Galea, L.A. (2007) Hippocampus-Dependent Learning Promotes Survival of New Neurons in the Dentate Gyrus at a Specific Time during Cell Maturation. Neuroscience, 149, 273-285. https://doi.org/10.1016/j.neuroscience.2007.07.046

[26] Wolf, S.A., et al. (2006) Cognitive and Physical Activity Differently Modulate Disease Progression in the Amyloid Precursor Protein (APP)-23 Model of Alzheimer's Disease. Biological Psychiatry, 60, 1314-1323. https://doi.org/10.1016/j.biopsych.2006.04.004

[27] Kempermann, G., Kuhn, H.G. and Gage, F.H. (1997) More Hippocampal Neurons in Adult Mice Living in an Enriched Environment. Nature, 386, 493-495. https://doi.org/10.1038/386493a0

[28] Kempermann, G., Gast, D. and Gage, F.H. (2002) Neuroplasticity in Old Age: Sustained Fivefold Induction of Hippocampal Neurogenesis by Long-Term Environmental Enrichment. Annals of Neurology, 52, 135-143. https://doi.org/10.1002/ana.10262

[29] Segovia, G., Yagüe, A.G., García-Verdugo, J.M. and Mora, F. (2006) Environmental Enrichment Promotes Neurogenesis and Changes the Extracellular Concentrations of Glutamate and GABA in the Hippocampus of Aged Rats. Brain Research Bulletin, 70, 8-14. https://doi.org/10.1016/j.brainresbull.2005.11.005

[30] Pham, T.M., et al. (1999) Changes in Brain Nerve Growth Factor Levels and Nerve Growth Factor Receptors in Rats Exposed to Environmental Enrichment for One Year. Neuroscience, 94, 279-286. https://doi.org/10.1016/S0306-4522(99)00316-4

[31] Young, D., Lawlor, P.A., Leone, P., Dragunow, M. and During, M.J. (1999) Environmental Enrichment Inhibits Spontaneous Apoptosis, Prevents Seizures and Is 
Neuroprotective. Nature Medicine, 5, 448-453. https://doi.org/10.1038/7449

[32] Cao, L., et al. (2004) VEGF Links Hippocampal Activity with Neurogenesis, Learning and Memory. Nature Genetics, 36, 827-835. https://doi.org/10.1038/ng1395

[33] Pham, T.M., et al. (2002) Environmental Influences on Brain Neurotrophins in Rats. Pharmacology Biochemistry \& Behavior, 73, 167-175. https://doi.org/10.1016/S0091-3057(02)00783-9

[34] Klein, S.L., Lambert, K.G., Durr, D., Schaefer, T. and Waring, R.E. (1994) Influence of Environmental Enrichment and Sex on Predator Stress Response in Rats. Physiology \& Behavior, 56, 291-297. https://doi.org/10.1016/0031-9384(94)90197-X

[35] Benaroya Milshtein, N., et al. (2015) Environmental Enrichment in Mice Decreases Anxiety, Attenuates Stress Responses and Enhances Natural Killer Cell Activity. European Journal of Neuroscience, 20, 1341-1347. https://doi.org/10.1111/j.1460-9568.2004.03587.x

[36] Williams, B.M., et al. (2001) Environmental Enrichment: Effects on Spatial Memory and Hippocampal CREB Immunoreactivity. Physiology \& Behavior, 73, 649-658. https://doi.org/10.1016/S0031-9384(01)00543-1

[37] Zhong, L., et al. (2007) Preweaning Exposure to Enriched Environment Induces Hippocampal Neurogenesis: Experiment with Rats. National Medical Journal of China, 87, 1559.

[38] Zhong, L., et al. (2009) Calmodulin Activation Is Required for the Enhancement of Hippocampal Neurogenesis Following Environmental Enrichment. Neurological Research, 31, 707-713. https://doi.org/10.1179/174313209X380856

[39] Bröcher, S., Artola, A. and Singer, W. (1992) Agonists of Cholinergic and Noradrenergic Receptors Facilitate Synergistically the Induction of Long-Term Potentiation in Slices of Rat Visual Cortex. Brain Research, 573, 27-36. https://doi.org/10.1016/0006-8993(92)90110-U

[40] Blennow, K., de Leon, M.J. and Zetterberg, H. (2006) Alzheimer's Disease. The Lancet, 368, 387-403. https://doi.org/10.1016/S0140-6736(06)69113-7

[41] Patterson, C. (2018) World Alzheimer Report 2018. The State of the Art of Dementia Research: New Frontiers. An Analysis of Prevalence, Incidence, Cost and Trends. Alzheimer's Disease International.

[42] Yong, J.I., et al. (2014) A 200-Year History of Alzheimer's Disease. Chinese Journal of Contemporary Neurology \& Neurosurgery, 14, 156-160.

[43] Ge, R. (2014) Progress in the Researches of Alzeimer's Disease. Chinese and Foreign Medical Research, 9, 155-157.

[44] De Felice, F.G. (2013) Alzheimer's Disease and Insulin Resistance: Translating Basic Science into Clinical Applications. Journal of Clinical Investigation, 123, 531-539. https://doi.org/10.1172/JCI64595

[45] Selkoe, D.J. (2001) Alzheimer's Disease: Genes, Proteins, and Therapy. Physiological Reviews, 81, 741-766. https://doi.org/10.1152/physrev.2001.81.2.741

[46] Torreilles, F. and Touchon, J. (2002) Pathogenic Theories and Intrathecal Analysis of the Sporadic form of Alzheimer's Disease. Progress in Neurobiology, 66, 191-203. https://doi.org/10.1016/S0301-0082(01)00030-2

[47] Lee, H.G., et al. (2004) Challenging the Amyloid Cascade Hypothesis: Senile Plaques and Amyloid-Beta as Protective Adaptations to Alzheimer Disease. Annals of the New York Academy of Sciences, 1019, 1-4. https://doi.org/10.1196/annals.1297.001

[48] Zhang, W., et al. (2012) Multiple Inflammatory Pathways Are Involved in the Development and Progression of Cognitive Deficits in APPswe/PS1dE9 Mice. Neuro- 
biology of Aging, 33, 2661-2677.

https://doi.org/10.1016/j.neurobiolaging.2011.12.023

[49] Sondag, C.M. and Combs, C.K. (2010) Amyloid Precursor Protein Cross-Linking Stimulates Beta Amyloid Production and Pro-Inflammatory Cytokine Release in Monocytic Lineage Cells. Journal of Neurochemistry, 97, 449-461. https://doi.org/10.1111/j.1471-4159.2006.03759.x

[50] Streit, W.J. (2010) Microglia and Alzheimer's Disease Pathogenesis. Journal of Neuroscience Research, 77, 1-8. https://doi.org/10.1002/jnr.20093

[51] Tweedie, D., et al. (2012) Tumor Necrosis Factor- $\alpha$ Synthesis Inhibitor 3,6'-Dithiothalidomide Attenuates Markers of Inflammation, Alzheimer Pathology and Behavioral Deficits in Animal Models of Neuroinflammation and Alzheimer's Disease. Journal of Neuroinflammation, 9, 106.

https://doi.org/10.1186/1742-2094-9-106

[52] Eikelenboom, P., et al. (2012) Whether, When and How Chronic Inflammation Increases the Risk of Developing Late-Onset Alzheimer's Disease. Alzheimer's Research \& Therapy, 4, 15. https://doi.org/10.1186/alzrt118

[53] Wallin, Å.K., Blennow, K., Andreasen, N. and Minthon, L. (2006) CSF Biomarkers for Alzheimer's Disease: Levels of $\beta$-Amyloid, Tau, Phosphorylated Tau Relate to Clinical Symptoms and Survival. Dementia \& Geriatric Cognitive Disorders, 21, 131-138. https://doi.org/10.1159/000090631

[54] Tapiola, T., et al. (1997) The Level of Cerebrospinal Fluid Tau Correlates with Neurofibrillary Tangles in Alzheimer's Disease. NeuroReport, 8, 3961-3963.

https://doi.org/10.1097/00001756-199712220-00022

[55] Verdier, Y., Zarándi, M. and Penke, D.B. (2004) Amyloid $\beta$-Peptide Interactions with Neuronal and Glial Cell Plasma Membrane: Binding Sites and Implications for Alzheimer's Disease. Journal of Peptide Science, 10, 229-248. https://doi.org/10.1002/psc.573

[56] Ittner, L.M. and Götz, J. (2010) Amyloid- $\beta$ and Tau-A Toxic Pas de Deux in Alzheimer's Disease. Nature Reviews Neuroscience, 12, 65-72.

[57] Amadoro, G., et al. (2011) Endogenous A $\beta$ Causes Cell Death via Early Tau Hyperphosphorylation. Neurobiology of Aging, 32, 969-990. https://doi.org/10.1016/j.neurobiolaging.2009.06.005

[58] Zhang, S.J., et al. (2012) Amyloid- $\beta$ and Tau in Alzheimer's Disease. Chemistry of Life, 32, 254-258.

[59] Smith, M.A., Zhu, X., et al. (2010) Increased Iron and Free Radical Generation in Preclinical Alzheimer Disease and Mild Cognitive Impairment. Journal of Alzheimer's Disease, 19, 363-372. https://doi.org/10.3233/JAD-2010-1239

[60] Perry, G., et al. (2000) Oxidative Damage in Alzheimer's Disease: The Metabolic Dimension. International Journal of Developmental Neuroscience, 18, 417-421. https://doi.org/10.1016/S0736-5748(00)00006-X

[61] Liu, F., et al. (2015) The Oxidative Stress and Antioxidative Therapy in Alzheimer's Disease. Journal of Jinan University (Natural Science \& Medicine Edition), 36, 406-409.

[62] Li, L., Zhang, X. and Le, W. (2010) Autophagy Dysfunction in Alzheimer's Disease. Neurodegenerative Diseases, 7, 265-271. https://doi.org/10.1159/000276710

[63] Yoon, S.Y. and Kim, D.H. (2016) Alzheimer's Disease Genes and Autophagy. Brain Research, 1649, 201-209. https://doi.org/10.1016/j.brainres.2016.03.018

[64] Sanchez-Varo, R., et al. (2012) Abnormal Accumulation of Autophagic Vesicles 
Correlates with Axonal and Synaptic Pathology in Young Alzheimer's Mice Hippocampus. Acta Neuropathologica, 123, 53-70. https://doi.org/10.1007/s00401-011-0896-x

[65] Kim, S., et al. (2014) NDP52 Associates with Phosphorylated Tau in Brains of an Alzheimer Disease Mouse Model. Biochemical \& Biophysical Research Communications, 454, 196-201. https://doi.org/10.1016/j.bbrc.2014.10.066

[66] Schaeffer, V.R. and Goedert, M. (2012) Stimulation of Autophagy Is Neuroprotective in a Mouse Model of Human Tauopathy. Autophagy, 135, 2169-2177.

[67] Hamano, T., et al. (2010) Autophagic-Lysosomal Perturbation Enhances Tau Aggregation in Transfectants with Induced Wild-Type Tau Expression. European Journal of Neuroscience, 27, 1119-1130. https://doi.org/10.1111/j.1460-9568.2008.06084.x

[68] Peters, O. (2012) Alzheimer's Disease: Are Non-Steroidal Anti-Inflammatory Drugs Effective? Deutsche Medizinische Wochenschrift, 137, 2627.

[69] Menting, K.W. and Claassen, J.A. (2014) $\beta$-Secretase Inhibitor: A Promising Novel Therapeutic Drug in Alzheimer's Disease. Frontiers in Aging Neuroscience, 6, 165. https://doi.org/10.3389/fnagi.2014.00165

[70] Bozzali, M., et al. (2013) Brain Tissue Modifications Induced by Cholinergic Therapy in Alzheimer's Disease. Human Brain Mapping, 34, 3158-3167. https://doi.org/10.1002/hbm.22130

[71] Men, Y., et al. (2015) Advances in Active Constituents of Traditional Chinese Medicines for Treating Alzheimer's Disease. Letters in Biotechnology, 4, 587-590.

[72] Wang, Q. (2012) Advances in the Researches of Acetylcholinesterase Inhibitors in the Treatment of Alzheimer's Disease. Science \& Technology Information, 24, 127.

[73] Sevigny, J., et al. (2016) The Antibody Aducanumab Reduces A $\beta$ Plaques in Alzheimer's Disease. Nature, 537, 50-56. https://doi.org/10.1038/nature19323

[74] Congdon, E.E. and Sigurdsson, E.M. (2018) Tau-Targeting Therapies for Alzheimer Disease. Nature Reviews. Neurology, 14, 399-415.

https://doi.org/10.1038/s41582-018-0013-z

[75] Doody, R.S., et al. (2014) Phase 3 Trials of Solanezumab for Mild-to-Moderate Alzheimer's Disease. The New England Journal of Medicine, 370, 311-321. https://doi.org/10.1056/NEJMoa1312889

[76] Bohrmann, B., et al. (2012) Gantenerumab: A Novel Human Anti-A $\beta$ Antibody Demonstrates Sustained Cerebral Amyloid- $\beta$ Binding and Elicits Cell-Mediated Removal of Human Amyloid- $\beta$. Journal of Alzheimer's Disease, 28, 49-69. https://doi.org/10.3233/JAD-2011-110977

[77] Salloway, S., et al. (2014) Two Phase 3 Trials of Bapineuzumab in Mild-to-Moderate Alzheimer's Disease. The New England Journal of Medicine, 370, 322-333. https://doi.org/10.1056/NEJMoa1304839

[78] Colacurcio, D.J., Pensalfini, A., Jiang, Y. and Nixon, R.A. (2017) Dysfunction of Autophagy and Endosomal-Lysosomal Pathways: Roles in Pathogenesis of Down Syndrome and Alzheimer's Disease. Free Radical Biology \& Medicine, 114, 40-51. https://doi.org/10.1016/j.freeradbiomed.2017.10.001

[79] Li, Q., Liu, Y. and Sun, M. (2017) Autophagy and Alzheimer's Disease. Cellular \& Molecular Neurobiology, 37, 377-388. https://doi.org/10.1007/s10571-016-0386-8

[80] Gu, J., Congdon, E.E. and Sigurdsson, E.M. (2013) Two Novel Tau Antibodies Targeting the 396/404 Region Are Primarily Taken Up by Neurons and Reduce Tau Protein Pathology. Journal of Biological Chemistry, 288, 33081-33095. 
https://doi.org/10.1074/jbc.M113.494922

[81] Lei, W.H., et al. (2011) The Current Status and Prospect of Healing Garden. Chinese Landscape Architecture, 27, 31-36.

[82] Koike, H., et al. (1999) Membrane-Anchored Metalloprotease MDC9 Has an Alpha-Secretase Activity Responsible for Processing the Amyloid Precursor Protein. Biochemical Journal, 343, 371-375. https://doi.org/10.1042/bj3430371

[83] Wang, L., et al. (2014) Effects of Aluminium on $\beta$-Amyloid (1-42) and Secretases (APP-Cleaving Enzymes) in Rat Brain. Neurochemical Research, 39, 1338-1345. https://doi.org/10.1007/s11064-014-1317-Z

[84] Liu, H.L., Zhao, G., Zhang, H. and Shi, L.-D. (2013) Long-Term Treadmill Exercise Inhibits the Progression of Alzheimer's Disease-Like Neuropathology in the Hippocampus of APP/PS1 Transgenic Mice. Behavioural Brain Research, 256, 261-272. https://doi.org/10.1016/j.bbr.2013.08.008

[85] Nichol, K.E., et al. (2008) Exercise Alters the Immune Profile in Tg2576 Alzheimer Mice toward a Response Coincident with Improved Cognitive Performance and Decreased Amyloid. Journal of Neuroinflammation, 5, 13. https://doi.org/10.1186/1742-2094-5-13

[86] Garcíamesa, Y., et al. (2012) Melatonin plus Physical Exercise Are Highly Neuroprotective in the 3xTg-AD Mouse. Neurobiology of Aging, 33, 1124.e13-1124.e29.

[87] Adlard, P.A., Perreau, V.M., Pop, V. and Cotman, C.W. (2005) Voluntary Exercise Decreases Amyloid Load in a Transgenic Model of Alzheimer's Disease. Journal of Neuroscience, 25, 4217-4221. https://doi.org/10.1523/JNEUROSCI.0496-05.2005

[88] Yu, F., et al. (2014) Effects of Treadmill Exercise on $\beta$-Amyloid Precursor Protein and Tau Protein Gene Expressions in Hippocampus of D-Galactose Alzheimer's Disease Rats. Chinese Journal of Rehabilitation Medicine, 29, 1010-1015.

[89] Yu, F., et al. (2016) Research on Effect of $\alpha$-Secretase on Regulating Hippocampal APP and A $\beta$-42 in APP/PS1 Transgenic Mice of Alzeimer's Disease after Voluntary Wheel Running. China Sport Science, 36, 49-55.

[90] Kong, F.J., et al. (2013) Effect of Regular Aerobic Exercises on Learning and Memory of APP/PS1 Mice. Journal of Neuroscience and Mental Health, 13, 232-234.

[91] Marco, M., et al. (2014) Environmental Enrichment Strengthens Corticocortical Interactions and Reduces Amyloid- $\beta$ Oligomers in Aged Mice. Frontiers in Aging Neuroscience, 6, 1 .

[92] Maesako, M., et al. (2012) Environmental Enrichment Ameliorated High-Fat Diet-Induced A $\beta$ Deposition and Memory Deficit in APP Transgenic Mice. Neurobiology of Aging, 33, 1011.e11-1011.e23.

https://doi.org/10.1016/j.neurobiolaging.2011.10.028

[93] Li, J.Z., et al. (2015) Effects of Enriched Environment on Neurons Apoptosis in Hippocampal CA1 Region in Senescence Accelerated Mouse. Chinese Journal of Behavioral Medicine and Brain Science, 24, 113-116.

[94] Lazarov, O., et al. (2005) Environmental Enrichment Reduces A $\beta$ Levels and Amyloid Deposition in Transgenic Mice. Cell, 120, 701-713. https://doi.org/10.1016/j.cell.2005.01.015

[95] Hu, Y.S., et al. (2010) Complex Environment Experience Rescues Impaired Neurogenesis, Enhances Synaptic Plasticity, and Attenuates Neuropathology in Familial Alzheimer's Disease-Linked APPswe/PS1 $\triangle \mathrm{E} 9$ Mice. The FASEB Journal, 24, 1667-1681. https://doi.org/10.1096/fj.09-136945

[96] Lahianicohen, I., et al. (2011) Moderate Environmental Enrichment Mitigates Tau- 
opathy in a Neurofibrillary Tangle Mouse Model. Journal of Neuropathology and Experimental Neurology, 70, 610-621. https://doi.org/10.1097/NEN.0b013e318221bfab

[97] Leem, Y.H., et al. (2010) Repression of Tau Hyperphosphorylation by Chronic Endurance Exercise in Aged Transgenic Mouse Model of Tauopathies. Journal of Neuroscience Research, 87, 2561-2570. https://doi.org/10.1002/jnr.22075

[98] Kaytor, M.D. and Orr, H.T. (2002) The GSK3 $\beta$ Signaling Cascade and Neurodegenerative Disease. Current Opinion in Neurobiology, 12, 275-278. https://doi.org/10.1016/S0959-4388(02)00320-3

[99] Fan, D., Li, J., Zheng, B., Hua, L. and Zuo, Z. (2016) Enriched Environment Attenuates Surgery-Induced Impairment of Learning, Memory, and Neurogenesis Possibly by Preserving BDNF Expression. Journal of Molecular Neurobiology, 53, 344-354. https://doi.org/10.1007/s12035-014-9013-1

[100] Ziegler-Waldkirch, S., Marksteiner, K., Stoll, J., et al. (2018) Environmental Enrichment Reverses A $\beta$ Pathology during Pregnancy in a Mouse Model of Alzheimer's Disease. Acta Neuropathologica Communications, 6, 44. https://doi.org/10.1186/s40478-018-0549-6

[101] Xu, H., et al. (2016) Environmental Enrichment Potently Prevents Microglia-Mediated Neuroinflammation by Human Amyloid $\beta$-Protein Oligomers. Journal of Neuroscience, 36, 9041-9056. https://doi.org/10.1523/JNEUROSCI.1023-16.2016

[102] Ambrée, O., et al. (2006) Reduction of Amyloid Angiopathy and A $\beta$ Plaque Burden after Enriched Housing in TgCRND8 Mice: Involvement of Multiple Pathways. American Journal of Pathology, 169, 544-552. https://doi.org/10.2353/ajpath.2006.051107

[103] Zhou, C.J., et al. (2017) Effect of Enriched Environment on Cognitive Function in Patients with Early and Middle-Aged Dementia. Psychological Doctor, 23, 8.

[104] Ying, X. (2017) Effect of Comprehensive Rehabilitation Training on Cognitive Function and Quality of Life in Patients with Alzheimer's Disease. Chinese Journal of Rural Medicine and Pharmacy, 24, 76-77.

[105] Zhang, J., et al. (2015) Effect of Comprehensive Rehabilitation Training on Cognitive Function and Daily Living Ability of Patients with Alzheimer's Disease. Shanxi Medical Journal, 44, 785-787.

[106] Van Dellen, A., Blakemore, C., Deacon, R., York, D. and Hannan, A.J. (2000) Delaying the Onset of Huntington's in Mice. Nature, 404, 721-722. https://doi.org/10.1038/35008142

[107] Lazic, S.E., et al. (2010) Neurogenesis in the R6/1 Transgenic Mouse Model of Huntington's Disease: Effects of Environmental Enrichment. European Journal of Neuroscience, 23, 1829-1838. https://doi.org/10.1111/j.1460-9568.2006.04715.x

[108] Frick, K.M. and Fernandez, S.M. (2003) Enrichment Enhances Spatial Memory and Increases Synaptophysin Levels in Aged Female Mice. Neurobiology of Aging, 24, 615-626. https://doi.org/10.1016/S0197-4580(02)00138-0

[109] Gelfo, F., Cutuli, D., Foti, F., et al. (2011) Enriched Environment Improves Motor Function and Increases Neurotrophins in Hemicerebellar Lesioned Rats. Neurorehabil Neural Repair, 25, 243-252. https://doi.org/10.1177/1545968310380926

[110] Prusky, G.T., Reidel, C. and Douglas, R.M. (2000) Environmental Enrichment from Birth Enhances Visual Acuity but Not Place Learning in Mice. Behavioural Brain Research, 114, 11-15. https://doi.org/10.1016/S0166-4328(00)00186-8 
[111] Nithianantharajah, J., Levis, H. and Murphy, M. (2004) Environmental Enrichment Results in Cortical and Subcortical Changes in Levels of Synaptophysin and PSD-95 Proteins. Neurobiology of Learning \& Memory, 81, 200-210. https://doi.org/10.1016/j.nlm.2004.02.002

[112] Chen, J. (2008) Noninvasive Study on Brain Neurotransmitter in the Patients with Alzheimer's Disease. Guangzhou Medical Journal, 39, 7-9.

[113] Pu, Z.X., et al. (2007) Influence of Environmental Enrichment at Different Stages of Development on the Expression of p38 in Hippocampus of Hypoxia-Ischemic Brain Damaged Rats. Chinese Journal of Child Health Care, 15, 632-634.

[114] Dezsi, G., et al. (2016) Environmental Enrichment Imparts Disease-Modifying and Transgenerational Effects on Genetically-Determined Epilepsy and Anxiety. Neurobiology of Disease, 93, 129-136. https://doi.org/10.1016/j.nbd.2016.05.005

[115] Crews, L., et al. (2008) Alpha-Synuclein Alters Notch-1 Expression and Neurogenesis in Mouse Embryonic Stem Cells and in the Hippocampus of Transgenic Mice. Journal of Neuroscience, 28, 4250-4260. https://doi.org/10.1523/JNEUROSCI.0066-08.2008

[116] Steiner, B., et al. (2006) Enriched Environment Induces Cellular Plasticity in the Adult Substantia Nigra and Improves Motor Behavior Function in the 6-OHDA Rat Model of Parkinson's Disease. Experimental Neurology, 199, 291-300. https://doi.org/10.1016/j.expneurol.2005.11.004

[117] Xie, H.Y., et al. (2011) New Progress in the Application of Enriched Environment in Neurodegenerative Diseases. Chinese Journal of Rehabilitation Medicine, 26, 592-596. 


\section{Abbreviations}

$\mathrm{A} \beta$ : Amyloid- $\beta$

AD: Alzheimer's Disease

APP: Amyloid Precursor Protein

IL-6: Interleukin-6

IL- $1 \beta$ : Interleukin- $1 \beta$

TNF- $\alpha$ : Tumor Necrosis Factor- $\alpha$

NSAIDs: Non-Steroidal Anti-Inflammatory Drugs

CREBcAMP: Response Element Binding Protein

Akt/PKB: Protein Kinase B

PI3: Kphosphatidylinositol kinase-3

NAP: Non-Amyloidogic Pathway

AP: Amyloidogic Pathway

SPs: Senile Plaques

EE: Enriched Environment

SE: Standard Environment

Drp1: Dynamin related protein 1

Caspases: cysteine proteolytic enzymes

p-Tauhyper: phosphorylated Tau protein

NFTs: Neurofibrillary Tangles

$\alpha$-sAPP: soluble APP

RNS: Reactive Nitrogen Species

ROS: Reactive Oxygen Species 